\title{
A Quantitative Survey of Conidiation Mutants in Aspergillus nidulans
}

\author{
By SYLVIA D. MARTINELLI* AND A. J. CLUTTERBUCK \\ Department of Genetics, University of Glasgow, Glasgow
}

(Accepted for publication 30 August I97I)

\begin{abstract}
SUMMARY
A quantitative survey has been made of conidiation mutants in Aspergillus nidulans. Conidiation mutants were 6 to 13 times more numerous than auxotrophic mutants, but growth tests showed that the mutant defect in $85 \%$ of the conidiation mutants was not confined to conidiation. The number of loci specifically involved in conidiation was estimated to be 45 to I 50. Mutants were classified according to the stage of the developmental block and also into asporogenous and oligosporogenous types; $64 \%$ were oligosporogenous, but in further tests, $84 \%$ of the asporogenous mutants were found to be temperature sensitive or slightly leaky, suggesting that many of the loci involved act only as modifiers in conidiation. The largest group of mutants was blocked before conidiophore formation, while many others failed at ill-defined stages of conidiation, suggesting that failure was due to the gradual build-up of metabolic deficiencies. A class of conidial maturation mutants has also been identified.
\end{abstract}

\section{INTRODUCTION}

Balassa (1969), in an extensive quantitative survey of sporulation mutants of Bacillus subtilis, concluded that about 800 genes were concerned in the sporulation process. Aspergillus nidulans is a much more complex organism than $B$. subtilis and yet Clutterbuck (I969) was able to find only two genes essential for the later stages of conidiation. Hopwood, Wildermuth \& Palmer (1970) have also found relatively few genes which control the production of sporulation septa in Streptomyces coelicolor. To investigate this apparent discrepancy, Balassa's procedure of comparing the numbers of auxotrophic and sporulation mutants has been applied to $A$. nidulans.

Balassa used both visual and replication techniques for the identification of sporulation mutants. Only about Io to I $5 \%$ of sporulation mutants were found to be totally asporogenous, the rest produced a genetically determined low frequency of spores and were termed oligosporogenous. Similarly, in the present work, conidiation mutants of Aspergillus nidulans have been identified by two criteria: the loss or alteration of the wild-type green spore colour, or failure of the mutants to be replicated by velvet. Oligosporogenous and asporogenous mutants have also been distinguished.

An additional basis of classification of sporulation mutants was employed by Ryter, Schaeffer \& Ionesco (I966), who defined seven stages of sporulation in Bacillus subtilis. A comparable series of stages for conidiation in Aspergillus nidulans has been put forward and used for the classification of mutants.

* Present address: Department of Genetics, Cambridge University, Cambridge. 


\section{METHODS}

General methods were those of Pontecorvo, Roper, Hemmons, Macdonald \& Bufton (1953). Incubation was normally at $37^{\circ}$, or at $22^{\circ}$ for temperature sensitive mutants.

Strains. The biAI (biotin requiring) strain was obtained from the Glasgow stocks of Aspergillus nidulans.

Media. Minimal medium (MM) was Czapek-Dox with I \% glucose and I $25 \%$ agar. Complete medium (CM) was made by the addition of the following to MM: peptone, $2 \mathrm{~g}$./1.; hydrolysed casein, $1.5 \mathrm{~g} . / 1$; yeast extract, $0.5 \mathrm{~g}$./1.; biotin, $2 \mu \mathrm{g}$./1.; p-aminobenzoic acid, $100 \mu \mathrm{g}$. $/ 1$.; pyridoxine and aneurin, $500 \mu \mathrm{g} . / 1$; nicotinamide and riboflavin, $1000 \mu \mathrm{g} . / 1$.

Mutagenesis. Conidia from 2-day-old colonies were suspended in Tween 80 and trismaleic buffer at pH 9.0, 0.05 M (Delić, Hopwood \& Friend, 1970) to which approximately $0.5 \mathrm{mg}$. $/ \mathrm{ml}$. (Expt I) or $0.1 \mathrm{mg} . / \mathrm{ml}$. (Expt II) of $N$-methyl- $N^{\prime}$-nitro- $N$-nitrosoguanidine (NTG) was added. The mixture was incubated at $37^{\circ}$ for $6 \mathrm{~h}$. in a shaking water bath; by this time all the NTG had decomposed so that the spores did not need washing. Suitable dilutions of treated conidia were plated on CM plus $0.8 \%$ sodium deoxycholate (Mackintosh \& Pritchard, 1963) to yield about 200 colonies per plate (Expt I) or 50 colonies per plate (Expt II). Plates were incubated for 3 days at $37^{\circ}$.

Classification of colonies. All colonies differing in appearance from the green wild-type were scored visually as sporulation mutants. They could then be subdivided into those affected in pigmentation only and those affected in various steps in spore formation. Colonies differing from the original strain in growth rate only were ignored. Mutants were also classified as poor or non-replicators by velvet replication on to fresh plates of $\mathrm{CM}+$ sodium deoxycholate, and as auxotrophs by replication on to $\mathrm{MM}+$ biotin + sodium deoxycholate. These replicas were examined after $16 \mathrm{~h}$. incubation.

Thioxanthine resistant mutants were classified after velvet replication on to $\mathrm{MM}$ containing $1.7 \mathrm{~mm}$-ammonium tartrate as a nitrogen source and $600 \mu \mathrm{M}$ 2-thioxanthine. Resistant mutants appeared green whereas normal colonies produced yellow conidia on this medium (Alderson \& Scazzocchio, 1967).

Growth measurement. Mutants were purified and the change in diameter of colonies from point inocula over a period of $64 \mathrm{~h}$. was compared with that of the biaAI strain.

\section{RESULTS}

Frequency of conidiation mutants. Conidia plated on CM and sodium deoxycholate after two different NTG treatments gave widely differing survival rates (Table I) but a large proportion of the survivors were, in both cases, conidiation mutants.

The number of loci concerned with condiation has been calculated by comparing the frequency of conidiation mutants with the frequency of auxotrophic mutants, for which the number of controlling loci can be estimated. The number of auxotrophic loci listed by Dorn (1967) in his review was 6r. This has been used in the calculation of Balassa (1969):

$\frac{\text { frequency of conidiation mutants }}{\text { frequency of auxotrophic mutants }} \times$ number of auxotrophic loci $=$ number of conidiation loci.

The ratio of conidiation mutants (classified visually or by replication) to auxotrophic mutants was found by experiment (Table I) to vary between 5.7: I and I3.3: I. This leads to an estimate of between 300 and 800 genes concerned with conidiation.

Alternative estimates of the number of conidiation loci, shown in Table 2, use the same 
calculation as previously but are based on the relative numbers of phenotypes due to mutation at other identifiable loci. These calculations give estimates ranging from 34 to I000 genes controlling conidiation.

Table I. Conidiation and auxotrophic mutants obtained after mutagen treatment of Aspergillus nidulans

\begin{tabular}{|c|c|c|c|c|c|c|c|c|}
\hline \multirow{2}{*}{$\begin{array}{l}\text { Experi- } \\
\text { ment* }\end{array}$} & \multirow{2}{*}{$\begin{array}{l}\text { Colonies } \\
\text { examined }\end{array}$} & \multirow{2}{*}{$\begin{array}{c}\text { Survival } \\
(\%)\end{array}$} & \multirow{2}{*}{$\begin{array}{c}\text { A } \\
\text { Auxo- } \\
\text { trophs } \\
\text { among } \\
\text { survivors } \\
(\%)\end{array}$} & \multirow{2}{*}{$\begin{array}{c}\text { B } \\
\text { Conidial } \\
\text { mutants } \\
\text { among } \\
\text { survivors } \\
\text { (visual } \\
\text { classifica- } \\
\text { tion) } \\
(\%)\end{array}$} & \multirow{2}{*}{$\begin{array}{c}\mathrm{C} \\
\text { Non- } \\
\text { replicators } \\
\text { among } \\
\text { survivors } \\
\begin{array}{c}(\%) \\
\end{array}\end{array}$} & \multirow{2}{*}{$\begin{array}{c}\text { D } \\
\text { Poor } \\
\text { repli- } \\
\text { cators } \\
\text { among } \\
\text { survivors } \\
(\%)\end{array}$} & \multicolumn{2}{|c|}{ Ratio } \\
\hline & & & & & & & B:A & $(C+D): A$ \\
\hline I & $38,85 \mathrm{I}$ & $\begin{array}{c}0.03 \\
50.0\end{array}$ & $\begin{array}{l}I \cdot 8 \\
0 \cdot 75\end{array}$ & $\begin{array}{r}24.0 \\
0.5\end{array}$ & IO & 2.2 & $\begin{array}{l}13.3: I \\
12 \cdot 7: I\end{array}$ & $\begin{array}{r}5 \cdot 7: I \\
0 \cdot 0: 1\end{array}$ \\
\hline 11 & 9,025 & 500 & 075 & 9.5 & $4 \%$ & 20 & $12 \% .1$ & 100.1 \\
\hline
\end{tabular}

* Expt I, $0.5 \mathrm{mg} / \mathrm{ml}$. NTG; Expt II, 0. I mg./ml. NTG.

Table 2. Estimates of the number of conidiation loci in Aspergillus nidulans

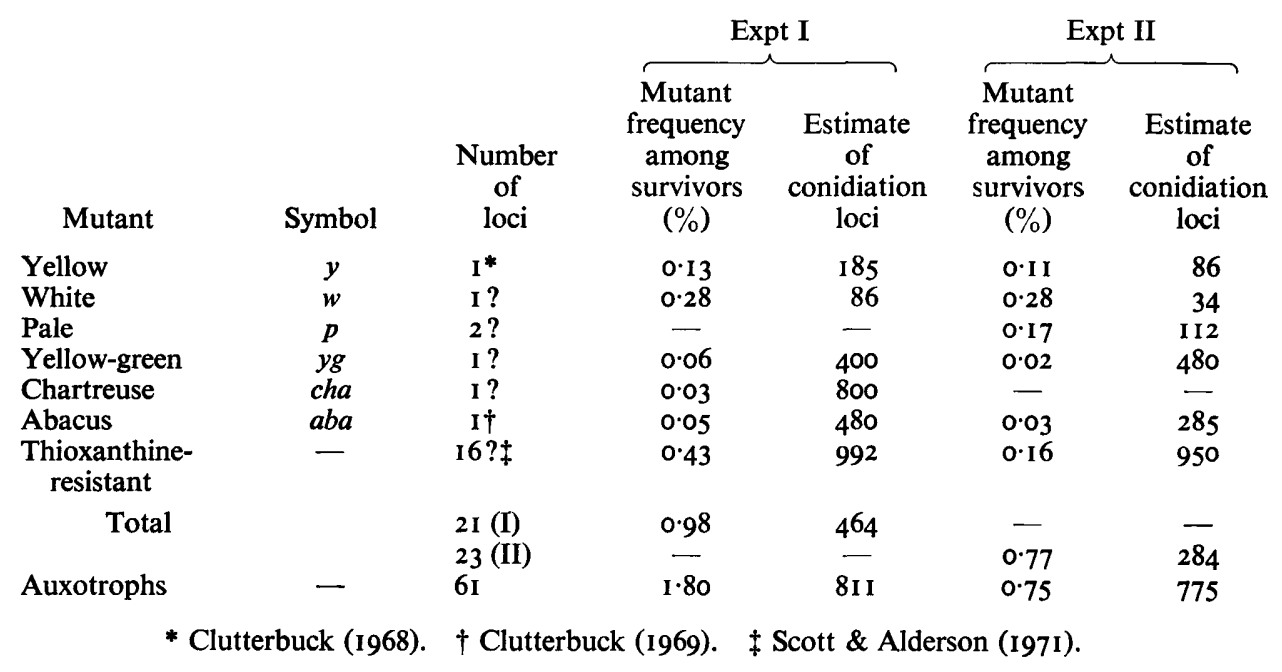

Frequency of slow-growing mutants. Many conidiation mutants were slow-growing. It can be argued that these mutants have a pleiotropic mutation affecting both vegetative growth and conidiation, or double mutations affecting the two characters separately. An estimate of the frequency of double mutants of this type was made by reference to yellow and white spore colour mutants from the same experiment. In previous experience, neither of these mutations affects growth, so that slow growers in this class should be due to double mutations. In Expt II, $10 \%$ of the spore colour mutants and $86.7 \%$ of the conidiation mutants were slow-growing. Using the figure of $10 \%$ to correct for double mutants among the slow-growing conidiation mutants,

$$
\frac{86.7 \%-10 \%}{100 \%-10 \%}=85.5 \%
$$

This is then the figure for mutants which are slow-growing solely as a result of pleiotropic mutations which affect both conidiation and growth. 
Frequency of different types of conidiation mutants. In Expt II, all conidiation mutants recognized visually or by replication were classified on the original plates, firstly into asporogenous and oligosporogenous classes and secondly according to the stage at which development fails (see Fig. I and Table 3).

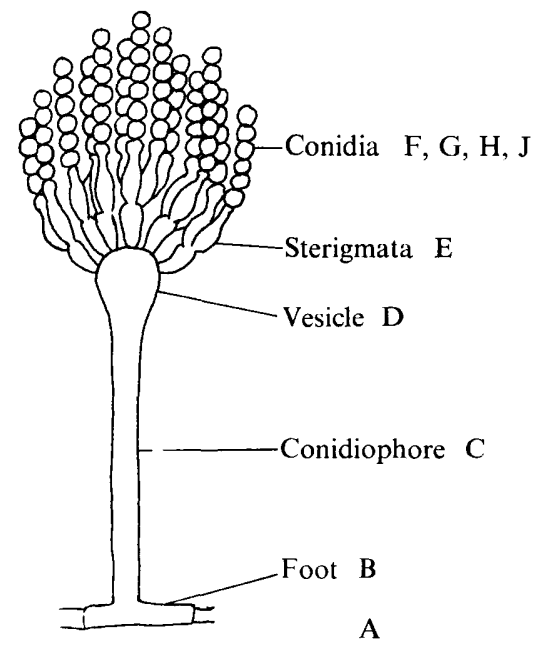

Fig. I. Diagram of wild-type conidial head of Aspergillus nidulans. Letters refer to the stages listed in Table 3 at which development is blocked in mutants.

Table 3. Numbers of mutants blocked at different stages of conidiation in Aspergillus nidulans

\begin{tabular}{|c|c|c|c|c|c|c|c|}
\hline $\begin{array}{l}\text { Bacillus } \\
\text { subtilis } \\
\text { equiva- } \\
\text { lent }\end{array}$ & $\begin{array}{l}\text { Mutant } \\
\text { group }\end{array}$ & $\begin{array}{l}\text { Stage of failure } \\
\text { of mutants }\end{array}$ & \multicolumn{2}{|c|}{ Oligosporogenous } & \multicolumn{3}{|c|}{ Asporogenous } \\
\hline 0 & A & $\left.\begin{array}{l}\text { Vegetative preparations } \\
\text { Foot cell differentiation }\end{array}\right\}$ & 'fluffy and 'flat' & 413 & 'fluffy' & and & 216 \\
\hline \multirow{3}{*}{ I, II } & $\mathrm{C}$ & Conidiophore development & stunted & o & & - & - \\
\hline & $\mathrm{D}$ & Vesicle formation & - & - & bristle & & I \\
\hline & E & Sterigmata formation & - & - & Various & & 12 \\
\hline III & $\mathrm{F}$ & Conidial bud formation & medusa & 0 & \multicolumn{2}{|l|}{ abacus } & 3 \\
\hline IV & G & Refractility & \multicolumn{2}{|c|}{ wet-white } & \multirow{3}{*}{$\begin{array}{l}\text { ance } \\
\text { arance }\end{array}$} & 0 & \\
\hline \multirow[t]{3}{*}{ VI } & $\mathbf{H}$ & Maturation & & & & 50 & \\
\hline & & & \multirow{2}{*}{\multicolumn{2}{|c|}{$\begin{array}{l}\text { Abnormal apr } \\
\text { Various }\end{array}$}} & & 34 & \\
\hline & $\mathbf{J}$ & Pigmentation & & & & 31 & \\
\hline
\end{tabular}

The data in this table are from Experiment II. Mutant types in italics correspond to loci described by Clutterbuck (1969).

For asporogenous mutants, classification by stage of failure could be done precisely, but the oligosporogenous mutants were difficult to assign to a particular stage since different conidiophores on the same colony developed to different extents. In such cases, the mutants are classified in Table 3 according to the earliest stage of development at which the defect is manifest.

By far the largest group contains those oligosporogenous mutants whose defect is first apparent at conidiophore initiation (stages $\mathbf{A}$ and $\mathrm{B}$ ). There are also a considerable number of asporogenous mutants at these stages. The majority of these have no conidiophores at all but some have a reduced number of conidiophores which, however, fail to bear any conidia. 
The other large group of mutants is the hitherto unrecognized class falling in group $\mathrm{H}$. Mutants of this type produce superficially normal or only slightly abnormal conidia which do not retain their viability. They are, therefore, detected by their failure to replicate. Only non-replicators of this type have been included in Table 3, poor replicators have been omitted.

Mutants with a normal number of conidiophores but failing at stages D to $F$ were relatively rare. They included one bristle and three abacus mutants (Clutterbuck, I969) as well as three other asporogenous mutants which can be discarded as they are slow-growing, and two oligosporogenous mutants.

stunted, medusa and wet-white Mutants (Clutterbuck, 1969) which mark distinctive stages shown in Table 3 were not found in Expt II, although a few were recognized in Expt I. dark Mutants were not scored in either experiment and ivory mutants would not be detected in this type of study.

Detailed analysis of asporogenous mutants. A sample of 98 mutants classified on the original mutation plates as asporogenous (stage A and B mutants) were purified and inoculated on CM plates (without sodium deoxycholate) for more detailed classification. Mutants classified as leaky produced a few conidia at $37^{\circ}$, while those described as temperature sensitive only produced conidia after transfer to $22^{\circ}$. Relatively few of the stage A and B mutants remained asporogenous on reclassification.

\section{DISCUSSION}

Factors affecting estimates of conidiation loci. Several factors influence the number of auxotrophic and conidiation mutants isolated by visual scoring and replica plating techniques. The 'complete' medium used in these experiments was deficient in several amino acids and vitamins, hence mutants for all these types of auxotrophic loci would not be isolated. The use of sodium deoxycholate in the medium might also affect the number of auxotrophic and conidiation mutants isolated. Therefore, in the calculation used in the results, only those loci at which it has been shown that mutants are readily recovered should be used. The list published by Dorn (1967) seems appropriate for this purpose.

Mutants have been isolated which can be cross-fed for normal growth requirements such as biotin (Pontecorvo et al. 1953) and for conidiation and conidial pigmentation (S. D. Martinelli, unpublished results). Consequently, both auxotrophic and conidiation mutants may be masked by a high density of plating, allowing repair by diffusion, between colonies. The relatively higher percentage of both types of mutant at the higher plating density used in Expt I (Table I) indicates that this has not played an important part in the numbers of mutants observed.

The difference between the number of conidiation mutants found by the two methods of classification can be accounted for by oligosporogenous mutants which normally replicate satisfactorily but which are recognized in the visual classification. There is also a class of mutants which are normal in appearance but fail to replicate. Some of these non-replicating normal mutants (group $\mathbf{H}$ in Table 3) may be defective in conidial maturation, while others appear to be heterokaryons between pairs of complementing lethals (A. J. Clutterbuck \& S. D. Martinelli, unpublished results).

Calculation of the number of conidiation loci. Using a calculation similar to Balassa (1969), an estimate of between 300 and 800 genes controlling conidiation has been made. A second series of calculations has also been made, in which the frequency of conidiation mutants isolated has been compared with the frequency of other easily recognizable phenotypes, 
due to mutation at identified loci. These give estimates of between 30 and 1000 genes affecting conidiation. An estimate of about 1000 genes comes from the comparison of the frequency of conidial mutants (visually scored) with the number of thioxanthine-resistant mutants. The latter group includes $\mathrm{I} 6$ loci with widely varying mutation rates, such as may be expected for different conidiation loci.

All the above estimates are concerned with genes in which mutation leads to a large or small effect on conidiation. Many of these mutants may have a defect expressing itself well before conidiation, such as pleiotropic mutations affecting both growth and conidiation. These mutants should be regarded as generally defective rather than specifically defective in conidiation. If the adjustment is made for slow-growing pleiotropic mutations, then $85.5 \%$ of the mutants can be discarded. The estimates for the number of loci involved specifically in conidiation can then be revised to $45-150$.

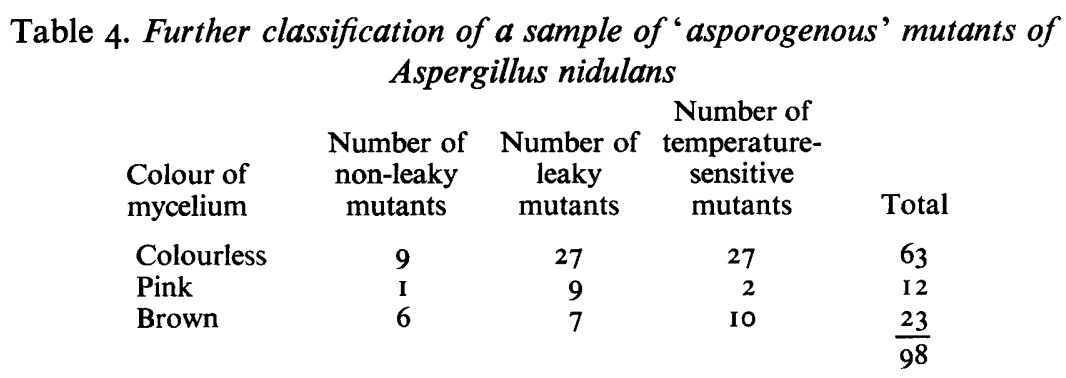

The classification and mode of action of conidiation mutants. Sixty-four per cent of the mutants blocked before conidium formation were classified on the original plates as oligosporogenous (Table 3) and $84 \%$ of these originally classified as asporogenous were later found to be leaky or temperature-sensitive (Table 4). Such a high proportion of oligosporogenous mutants in these experiments is comparable with Balassa's results for Bacillus subtilis.

There are two possible types of explanation for such leaky mutants; they may either have partial loss of activity at loci essential to conidiation or they may have complete (or partial) loss of activity at loci which merely modify the conidiation process. The frequency of partial mutants of the first type can be estimated by comparison with known loci: at the bristle, abacus and yellow (spore colour) loci partial mutants can readily be distinguished and were found by Clutterbuck (I968, I969) to form between I $8 \%$ and $22 \%$ of a total of I67 mutants. As seen above, the percentage of oligosporogenous mutants is very much higher than this, and the excess is probably made up of mutants of the second type, e.g. medusa mutants (Clutterbuck, 1969), in which the loci mutated are essentially modifiers. Mutation at such loci can only produce apparently leaky mutants rather than lead to a total loss of further development. The fact that so many of these mutants are temperature-sensitive suggests that the mutations are in loci whose functions are only essential at high temperatures.

In many of the mutants classified as oligosporogenous stage $A+B$ mutants, the reduction in the number of conidiophores was slight, but many of the conidiophores that were produced failed in development at subsequent stages. Such a pattern of abnormality suggests a gradual build-up of a metabolic defect which might be starvation for an essential component or the accumulation of a toxic product. A further feature of many of these mutants is that the conidiophore growth actually ceases rather than continuing in an aberrant form. This is in sharp distinction to the mutants studied by Clutterbuck (1969) in which failure is at a precise stage of development and is a failure of differentiation rather than growth. 
Where starvation or toxic accumulation affects hyphal growth as well as conidiation the mutants are not strictly regarded as conidiation mutants and can be excluded by a growth rate test. Many auxotrophs, for instance, when partially starved of their required nutrient, are nearly normal in growth but defective in conidiation. One mutant of this type is being studied further. This mutant requires for growth a diffusible substance, apparently of high molecular weight, that is excreted by normal conidiating colonies. At successively greater levels of the crossfeeding the mutant becomes fluffy, and then bears colourless conidia, and finally forms normal green conidia.

If metabolic mutants can be effectively excluded by growth rate tests, such a procedure should leave only mutants at loci which are inactive during vegetative growth but active during conidiation. A number of studies (reviewed by Turian, 1966; Murrell, 1967) have shown that in various organisms sporulation is accompanied by quantitative changes in enzyme levels. In such cases, unless conidiation specific isozymes are formed, the structural loci for these enzymes should not be regarded as conidiation loci, even though leaky mutants at such loci may fail to conidiate; on the other hand changes of enzyme levels may involve conidiation specific regulator loci.

In Bacillus subtilis one of the most conspicuous changes is the induction during sporulation of enzymes of the tricarboxylic cycle which are absent during vegetative growth (Fortnagel \& Freese, 1968). In fungi respiratory changes are less spectacular (Galbraith \& Smith, 1969), but may still be important. One of the types of oligosporogenous mutant, for instance, appears to show a shift from conidiation towards cleistothecium production, a shift that can be produced experimentally in cultures on agar by restricting the air supply (Pontecorvo et al. 1953).

Sporulation is induced in the majority of cells in Bacillus subtilis cultures by starvation conditions, especially exhaustion of the carbon and nitrogen sources (review: Mandelstam, 1969). It has been shown that glucose limitation is an effective inducer of conidiation in continuous liquid culture of Penicillium chrysogenum and nitrogen limitation in shaken liquid culture (Righelato, Trinci, Pirt \& Peat, 1968). In these circumstances the growth rate of conidiating mycelium was restricted to a rate at or near zero. Carter \& Bull (1969) also noted that cultures of Aspergillus nidulans, grown in stirred batch culture, conidiated after glucose exhaustion. In surface cultures the precise nutritional status of the conidiating region of a colony some distance behind the hyphal growth front is difficult to determine, but it is as likely to depend on the accumulation of metabolic products as on the supply of nutrients. Preliminary examination of some of the 'fluffy' mutants suggests that they produce excessive substrate mycelium as well as aerial mycelium. This feature, coupled with the invasive properties of fluffy mutants recently studied by Dorn (1970), indicate a failure of self-inhibition of hyphal growth in these mutants. In the wild-type this self-inhibition would conserve nutrients for conidiation, but in the mutants, the medium might be exhausted before conidiation could occur.

It is possible, on the other hand, that some mutants have lost the ability to conidiate in the presence of excess nutrients. It would be interesting therefore to test some oligosporogenous mutants defective in conidiophore initiation to see if any of them still conidiate under starvation conditions in submerged liquid culture. The very different nature of the two culture systems can be seen from their effect on a hyphal morphology mutant (Bainbridge \& Trinci, I969). In addition conidiation of submerged hyphae grown on agar under cellophane has been noted to depend on nitrogen or carbon source starvation (A. J. Clutterbuck, unpublished observations), so systems are clearly available in which the induction of conidiation by stimuli other than exposure to gaseous environments could be tested. By such tests, 
failures of conidial development might be separated from failures to respond to specific initiation stimuli.

One of us (S.D.M.) gratefully acknowledges the receipt of a Glasgow University Postdoctoral Research Fellowship during this work.

\section{REFERENCES}

Alderson, T. \& SCAzzocchio, C. (1967). A system for the study of inter-locus specificity for both forward and reverse mutation in at least eight gene loci in Aspergillus nidulans. Mutation Research 4, 567-577.

Bainbridge, B. W. \& Trinci, A. P. J. (1969). Colony and specific growth rates of normal and mutant strains of Aspergillus nidulans. Transactions of the British Mycological Society 53, 473-475.

BALASSA, G. (1969). Biochemical genetics of bacterial sporulation. I. Unidirectional pleiotropic interactions among genes controlling sporulation in Bacillus subtilis. Molecular and General Genetics 104, 73-I03.

CARTER, B. L. A. \& BulL, A. T. (1969). Studies of fungal growth and intermediary carbon metabolism under steady and non-steady state conditions. Biotechnology and Bioengineering II, 785-804.

Clutterbuck, A. J. (1968). New conidial colour mutants in Aspergillus nidulans. Aspergillus Newsletter 9, I4.

Clutterbuck, A. J. (1969). A mutational analysis of conidial development in Aspergillus nidulans. Genetics 63, 317-327.

Delić, V., Hopwood, D. A. \& Friend, E. J. (1970). Mutagenesis by $N$-methyl- $N$ '-nitro- $N$-nitrosoguanidine (NTG) in Streptomyces coelicolor. Mutation Research 9, I67-182.

DoRN, G. L. (1967). A revised map of the eight linkage groups of Aspergillus nidulans. Genetics 56, 619-63I.

DoRN, G. L. (1970). Genetic and morphological properties of undifferentiated and invasive variants of Aspergillus nidulans. Genetics 66, 267-279.

ForTNAGEL, P. \& Freese, E. (1968). Analysis of sporulation mutants. II. Mutants blocked in the citric acid cycle. Journal of Bacteriology 95, 143I-I438.

Galbraith, J. C. \& Sмith, J. E. (1969). Changes in activity of certain enzymes of the tricarboxylic acid cycle and the glyoxalate cycle during the initiation of conidiation of Aspergillus niger. Canadian Journal of Microbiology 15, 1207-1212.

Hopwood, D. A., Wildermuth, H. \& Palmer, H. M. (1970). Mutants of Streptomyces coelicolor defective in sporulation. Journal of General Microbiology 6r, 397-408.

Mackintosh, M. E. \& Pritchard, R. H. (1963). Production and replica plating of micro-colonies of Aspergillus nidulans. Genetical Research 4, 320-322.

MANDELSTAM, J. (1969). Regulation of bacterial spore formation. Symposium of the Society for General Microbiology 19, 377-40I.

Murrell, W. G. (1967). The biochemistry of the bacterial endospore. Advances in Microbial Physiology I, I33-25I.

Pontecorvo, G., Roper, J. A., Hemmons, L. M., Macdonald, K. D. \& Bufton, A. W. J. (I953). The genetics of Aspergillus nidulans. Advances in Genetics 5, 141-238.

Righelato, R. C., Trinci, A. P. J., Pirt, S. J. \& Peat, A. (I968). The influence of maintenance energy and growth rate on the metabolic activity, morphology and conidiation of Penicillium chrysogenum. Journal of General Microbiology 50, 399-4I 2.

Ryter, A., SChaeffer, P. \& IonesCo, H. (1966). Classification cytologique, par leur stade de blocage, des mutants de sporulation de Bacillus subtilis Marbug. Annals Institut Pasteur, Paris 110, 305-315.

SCOtT, B. R. \& ALDERSON, T. (197I). The random (non-specific) forward mutational response of gene loci in Aspergillus conidia after photosensitisation to near ultraviolet light $(365 \mathrm{~nm}$.) by 8-methoxypsoralen. Mutation Research 12, 29-34.

TuRIAN, G. (1966). Morphogenesis in ascomycetes. In The Fungi, vol. II, pp. 339-385. Edited by G. C. Ainsworth \& A. S. Sussman. New York \& London: Academic Press. 